\title{
Single-pulse and multipulse longitudinal phase space and temperature measurements of an intense ion beam
}

\author{
J. E. Coleman \\ Department of Nuclear Engineering, University of California at Berkeley, \\ 4155 Etcheverry Hall, MC 1730, Berkeley, California 94720, USA
}

\author{
P. A. Seidl, F. M. Bieniosek, M. A. Leitner, S. M. Lidia, J. L. Vay, and W. L. Waldron \\ Lawrence Berkeley National Laboratory, 1 Cyclotron Road, Berkeley, California 94720, USA \\ D. P. Grote \\ Lawrence Liveremore National Laboratory, Livermore, California 94550, USA
}

D. R. Welch

Voss Scientific, Albuquerque, New Mexico 87108, USA

(Received 21 November 2011; published 10 July 2012)

\begin{abstract}
Longitudinal phase space and temperature measurements were conducted on a 2-3 $\mu$ s long, singly charged $\mathrm{K}^{+}$ion bunch with an ion energy of $\sim 0.3 \mathrm{MeV}$ and current of $30 \mathrm{~mA}$. The principal objective of these experiments was to measure the longitudinal beam dynamics and study the limits of axial compression. The differences between the measured beam energy, longitudinal beam dynamics, and the amplitude and time history of the Marx voltage waveform were all quantified. Longitudinal phase space measurements indicate a slight chromaticity $(<1 \%)$ in the beam from head to tail. Record low longitudinal temperatures of $T_{z}=2-4 \times 10^{-2} \mathrm{eV}$ were measured for a beam bunch of this intensity with negligible effects from neutralizing the beam space charge with a background plasma. A qualitative comparison of experimental and calculated results are presented, which include time resolved longitudinal distributions, and phase space of the beam at $430 \mathrm{~cm}$.
\end{abstract}

PACS numbers: 52.59.Sa, 41.75.Ak, 41.85.Lc, 41.85.Ja

\section{INTRODUCTION}

Space-charge dominated ion beams used in accelerators to heat targets to warm dense matter (WDM) and drive fusion targets face a number of unique challenges. One of particular interest is the limit of axial compression and the longitudinal beam dynamics. The measured longitudinal temperature before acceleration and axial compression determines the energy spread, places an upper limit on axial compression, and constrains assumptions in computational models of the experiment. Minimal energy spread $\left(T_{z}\right)$ is a priority to minimize the acceleration schedule and maximize the beam intensity (current) on target.

Measurements of longitudinal phase space, temperature, and momentum spread have been made throughout the high energy physics community using several different techniques. Longitudinal phase space measurements were made with 16-MeV [1] and 250-MeV [2] electron beams using optical transition radiation. Each contained between $10^{9}$ and $10^{10}$ particles per bunch and had an energy spread

Published by the American Physical Society under the terms of the Creative Commons Attribution 3.0 License. Further distribution of this work must maintain attribution to the author(s) and the published article's title, journal citation, and DOI. that ranged from 1\%-9\%. Reference [3] reported a method of measuring the longitudinal phase space in a single shot by vertically streaking a $15-\mathrm{MeV}$ electron beam with two magnetic dipoles and an rf deflection cavity. The instrument has the ability to resolve a $\Delta E / E=2 \times 10^{-3}$.

Longitudinal temperature and momentum spread were measured using Schottky noise from two electrodes with a laser cooled, 40-keV $\mathrm{Mg}^{+}$ion beam [4] and an electron cooled, 70-GeV Au${ }^{79+}$ ion beam [5]. Reference [4] reported a $T_{\|}=5.9 \times 10^{-3} \mathrm{eV}$ and $\delta p / p=2.7 \times 10^{-4}$ initially with $10^{6}$ particles. After laser cooling, the particle count dropped to $N=3 \times 10^{4}$ due to residual gas scattering and the temperature dropped to $3.1 \times 10^{-4} \mathrm{eV}$ with a $\delta p / p=6.2 \times 10^{-5}$. Reference [5] reported a $\delta p / p=$ $5 \times 10^{-7}$ and $T_{\|}=4 \times 10^{-3} \mathrm{eV}$ for $N=4 \times 10^{3}$ particles. Beam position monitors and a $\delta p / p \sim N^{0.36}$ scaling law were used to estimate a $\delta p / p=2.5 \times 10^{-4}$ for an electron cooled, 2.61-GeV $\mathrm{Ar}^{18+}$ ion beam with $4 \times 10^{8}$ particles [6]. Authors in Ref. [4] reported a $T_{\|} \sim N^{0.3}$ dependence while authors in Ref. [5] reported a $\delta p / p \sim$ $N^{0.3}$ dependence, a slight discrepancy since $T_{\|} \sim(\delta p / p)^{2}$.

The design for a near-term ion accelerator, the NDCXII, capable of heating targets to $T_{e}>1 \mathrm{eV}$ is required to axially compress the beam from source to target by a factor $>500$. This will require a longitudinal temperature of 
$\ll 1 \mathrm{eV} . \mathrm{A} \mathrm{Li}^{+}$ion beam is extracted through an acceldecel diode at $\sim 100 \mathrm{kV}$ with a space-charge limited current $<100 \mathrm{~mA}$ and a pulse length near $500 \mathrm{~ns}$. This beam is injected into a solenoid matching section, after matching the space charge of the beam, it is accelerated and axially compressed using 12 induction cells to achieve a $1.2 \mathrm{MeV}$, $0.9 \mathrm{~ns}, 36 \mathrm{~A}$ pulse. This energy is slightly below the $1.9 \mathrm{MeV}$ Bragg peak, which is the required energy to optimize uniform heating through electronic stopping power at the center of the $\sim \mu \mathrm{m}$-thick $\mathrm{Al}$ target heated by a $\mathrm{Li}^{+}$ion driver [7-10].

The neutralized drift compression experiment (NDCX) at LBNL is exploring the physical limits of compression and focusing of ion beams and conducting preliminary WDM experiments [11-15]. In preparation for NDCX-II, the longitudinal phase space and temperature measurements described herein were used to quantitatively understand the requirements for the acceleration diode, acceleration schedule, and velocity tilt. An electrostatic dipole was used to make longitudinal phase space and temperature measurements. The number of particles in a bunch ranged from $4-6 \times 10^{11}$ ions/pulse. The measured momentum spread $\delta p / p$ of $2.03 \times 10^{-4}$ and longitudinal temperatures of $T_{z}=2-4 \times 10^{-2} \mathrm{eV}$ is on the same order as the accelerators mentioned above, with many orders of magnitude more particles and no active cooling. On a system level each of these accelerators have different transport physics, Refs. [1-3] are relativistic linear electron accelerators with picosecond bunch lengths, and Refs. [4-6] are storage rings with storage times on the order of seconds.

\section{EXPERIMENTAL SETUP}

An experimental configuration was used to study the limits of axial compression and the longitudinal dynamics of the $0.3-\mathrm{MeV}, 30-\mathrm{mA}$, singly charged $\mathrm{K}^{+}$ion beam used on the NDCX. An electrostatic energy analyzer (EEA) was added at the exit of the ferroelectric plasma source (FEPS) (Fig. 1) to measure the longitudinal phase space and temperature of the beam with and without plasma neutralization. The purpose of these measurements was to determine an upper limit on the axial compression and constrain previous assumptions of the longitudinal temperature before axial compression in computational models of the experiment.

The configuration of the focusing lattice used [Fig. 2(a)] was designed to project the beam waist as far downstream from the focusing lattice as possible, and as close to the

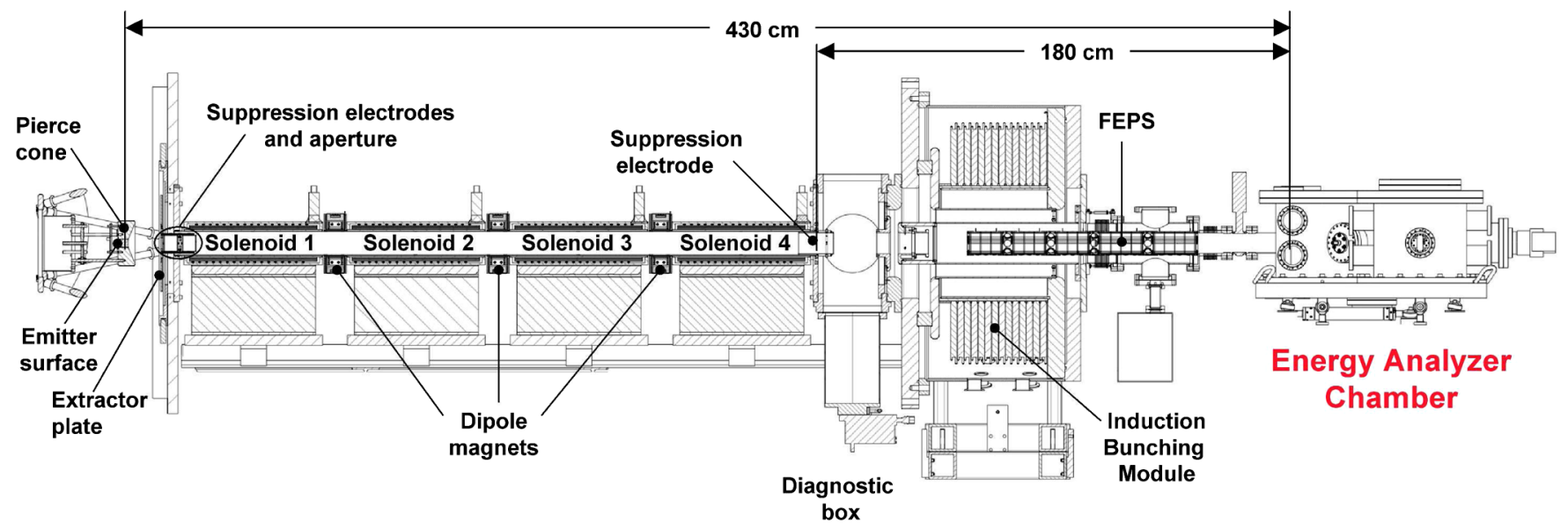

FIG. 1. Elevation view of the NDCX with the EEA added on at the exit of the FEPS.
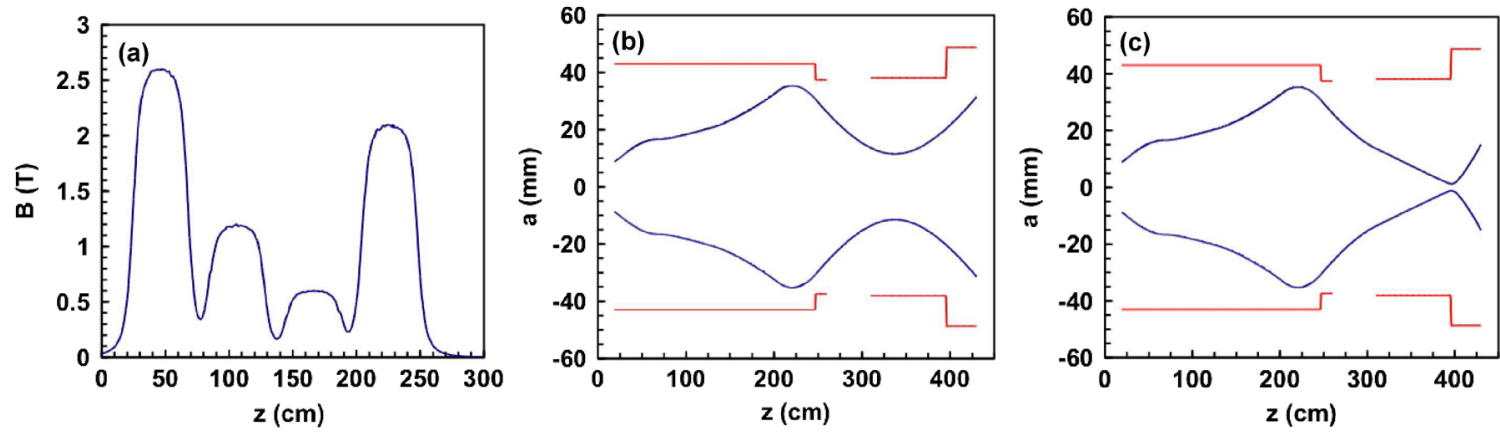

FIG. 2. (a) Axial magnetic field profile based on measurements; (b) calculated beam envelope for an unneutralized beam; and (c) calculated beam envelope for a beam neutralized by the FEPS. 
entrance of the EEA as possible. The purpose was to maximize the current density of the beam entering the spectrometer without scraping upstream. However, the current density must not be too high $\left(J>40 \mathrm{~A} / \mathrm{m}^{2}\right)$ because space-charge effects begin to play a role with $I>100 \mu \mathrm{A}$ in the spectrometer. The resulting beam envelope, shown in Fig. 2(b), safely transported the unneutralized beam to the entrance of the spectrometer without scraping and the neutralized case as well [Fig. 2(c)].

The transverse beam distribution was measured at the exit of the four-solenoid lattice $(z=262 \mathrm{~cm})$ to quantify if the transverse envelope parameters and centroid offsets were within the margin of error to avoid scraping. The measured horizontal beam envelope parameters were $a=25 \mathrm{~mm}$ and $a^{\prime}=-30 \mathrm{mrad}$ and centroid offsets were $\langle x\rangle=0.7 \mathrm{~mm}$ and $\left\langle x^{\prime}\right\rangle=2.5 \mathrm{mrad}$. The measured vertical beam envelope parameters were $b=25 \mathrm{~mm}$ and $b^{\prime}=-38 \mathrm{mrad}$ and centroid offsets $\langle y\rangle=-3 \mathrm{~mm}\left\langle y^{\prime}\right\rangle=$ $-2.7 \mathrm{mrad}$. Slight adjustments were made with steering dipoles to correct the centroid offsets to avoid beam scraping.

\section{SPECTROMETER}

Warren first presented a spectrometer, which is capable of measuring the longitudinal beam distribution, in 1947 [16]. The spectrometer consists of a cylindrical electrostatic dipole with a $90^{\circ}$ bend (Fig. 3).

The dimensions of the spectrometer are shown in Fig. 3. A dipole radius $a$ of $50 \mathrm{~cm}$ and the gap between the electrodes $d$ of $2.5 \mathrm{~cm}$ were chosen to measure up to a $1 \mathrm{MeV}$ beam. The corresponding energy on the dipole, $E$, for equal and opposite charge voltage on the plates, $V$, is

$$
E=\frac{V}{\ln \left(\frac{a+(d / 2)}{a-(d / 2)}\right)} .
$$

For this geometry $1 V=20 \mathrm{eV}$. The resolution $R$ of the spectrometer is defined by its geometry

$$
R=\frac{a(1+M)}{2 w M},
$$

where $w$ is the entrance slit width and $M$ is the magnification factor

$$
M=\frac{f}{l_{1}-g},
$$

where $f$ and $g$ are geometric factors

$$
\begin{aligned}
& f=\frac{a}{\sqrt{2}} \csc (\sqrt{2} \theta), \\
& g=\frac{a}{\sqrt{2}} \cot (\sqrt{2} \theta),
\end{aligned}
$$

and $\theta$ is the dipole angle, which in this case is $90^{\circ}$. Geometric factors $f$ and $g$ are also related by the object distance $l_{1}$ and image distance $l_{2}$ (Fig. 3):

$$
f^{2}=\left(l_{1}-g\right)\left(l_{2}-g\right) \text {. }
$$

$l_{1}$ and $l_{2}$ were chosen to be $17.52 \mathrm{~cm}$ based on the above equations and the chosen dipole radius and gap. The configuration used for measurements has the ability to resolve a beam distribution as narrow as $0.1 \mathrm{~mm}$ or $60 \mathrm{eV}$ for a $0.3-\mathrm{MeV}$ beam as shown in Table I. A $0.05-\mathrm{mm}$ wide slit could also be used to double the resolution.

This is the theoretical resolution; the actual resolution and absolute energy calibration of the diagnostic is dependent on several constraints. First, the mechanical tolerances and alignment of internal components must be aligned within $0.1 \mathrm{~mm}$ or better if possible. The high voltage on the dipole electrodes must be calibrated within $\Delta V / V=2 \times 10^{-4}$. Space-charge effects must be eliminated. Finally, beam and plasma loading, which can cause voltage drop on the electrodes, must be eliminated as well.

All of these specifications were met for our measurements. The DC voltage applied to each of the dipole electrodes was provided by an $85-\mathrm{kV}$, WR Series Glassman high voltage power supply [17]. This is a low

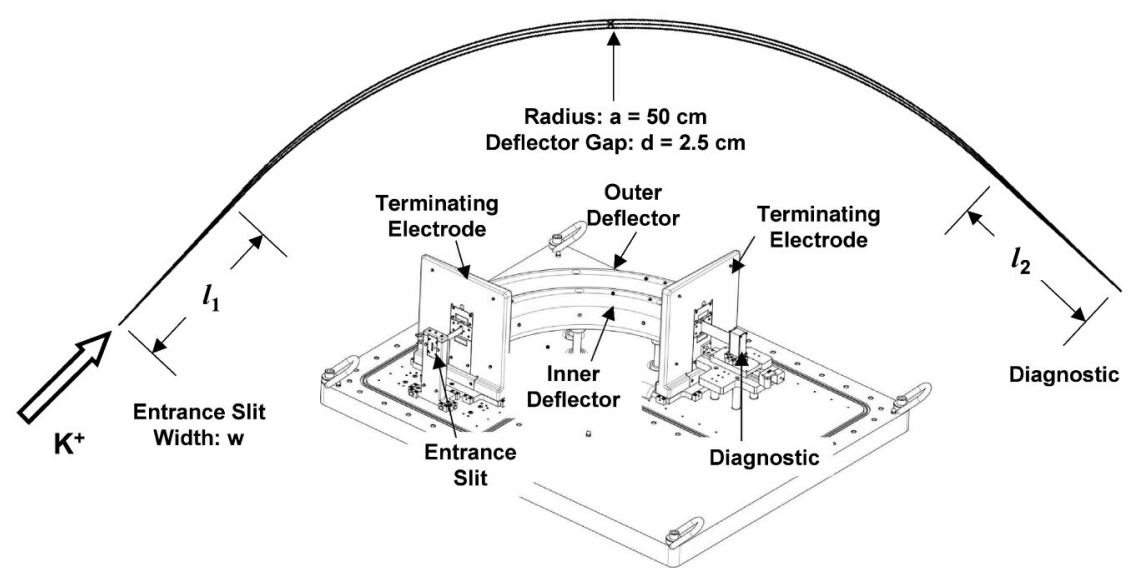

FIG. 3. Schematic of the electrostatic energy analyzer used on NDCX. 
TABLE I. Parameters for the electrostatic energy analyzer used on the NDCX.

\begin{tabular}{llcc}
\hline \hline Dipole angle & $\theta$ & $\circ$ & 90 \\
Dipole radius & $a$ & $\mathrm{~cm}$ & 50 \\
Dipole gap & $d$ & $\mathrm{~cm}$ & 2.5 \\
Geometric factor & $g$ & $\mathrm{~cm}$ & -26.9 \\
Geometric factor & $f$ & $\mathrm{~cm}$ & 44.4 \\
Object distance & $l_{1}$ & $\mathrm{~cm}$ & 17.52 \\
Image distance & $l_{2}$ & $\mathrm{~cm}$ & 17.52 \\
Entrance slit width & $w$ & $\mathrm{~mm}$ & 0.1 \\
Magnification & $M$ & & 1 \\
Resolution & $R$ & $1 \mathrm{part}$ per & 4921 \\
Energy scale & $E$ & $\mathrm{eV} / V$ & 20 \\
\hline \hline
\end{tabular}

ripple power supply that is regulated to better than $0.005 \%$. A Ross VD90-8.3Y-BD-LD-ALBD voltage divider was used to calibrate the output voltage from the power supplies to within $\pm 0.01 \%$ [18]. A capacitive coupling was used to monitor the voltage drop across a resistor. This was monitored at $50 \mathrm{MHz}$ with a Tektronix TDS3054 Series oscilloscope [19]. With this diagnostic we could monitor the image charges that were observed when the beam entered and exited the spectrometer and if any charge was collected on the plates due to scraping or beam loss $[13,14]$. Unless the voltage on the dipole was not set to steer the beam through there was negligible charge collected aside from the image currents.

There are additional constraints for absolute energy measurements. First, there are finite fringe fields at the beginning and end of the dipole bend (near the terminating electrodes). These fringe fields cause an effective increase or decrease in the dipole angle and this must be quantified. If the dipole bend is not exactly $90^{\circ}$ the beam will show up to the left or right of the expected transverse location on the focal plane. An additional check of the effective steering energy of the dipoles can be calibrated with a radiation source of known energy.

\section{ION TRAJECTORIES}

The unneutralized beam just upstream of the spectrometer had a radius of $31.5 \mathrm{~mm}$ and the beam neutralized by the FEPS had a radius of $23 \mathrm{~mm}$. The beam bunch passed through a $0.1 \mathrm{~mm}$ wide $\times 5 \mathrm{~mm}$ high slit plate (Fig. 4) which reduced the transmitted beam current from $30 \mathrm{~mA}$, which was incident on the slit plate, to $\sim 10 \mu \mathrm{A}$ for current densities $\sim 10-20 \mathrm{~A} / \mathrm{m}^{2}$, eliminating space-charge effects in the spectrometer. Although this is a reduction in signal $>3$ orders of magnitude, it is similar to our transverse phase space measurements $[13,14]$. The beam ribbon traversed the $90^{\circ}$ bend of the electrostatic dipole and was detected at the focal plane. The trajectory of ions of various energies through the spectrometer is illustrated in the schematic in Fig. 4. Ions with energy greater than the corresponding energy on the dipole will follow a larger

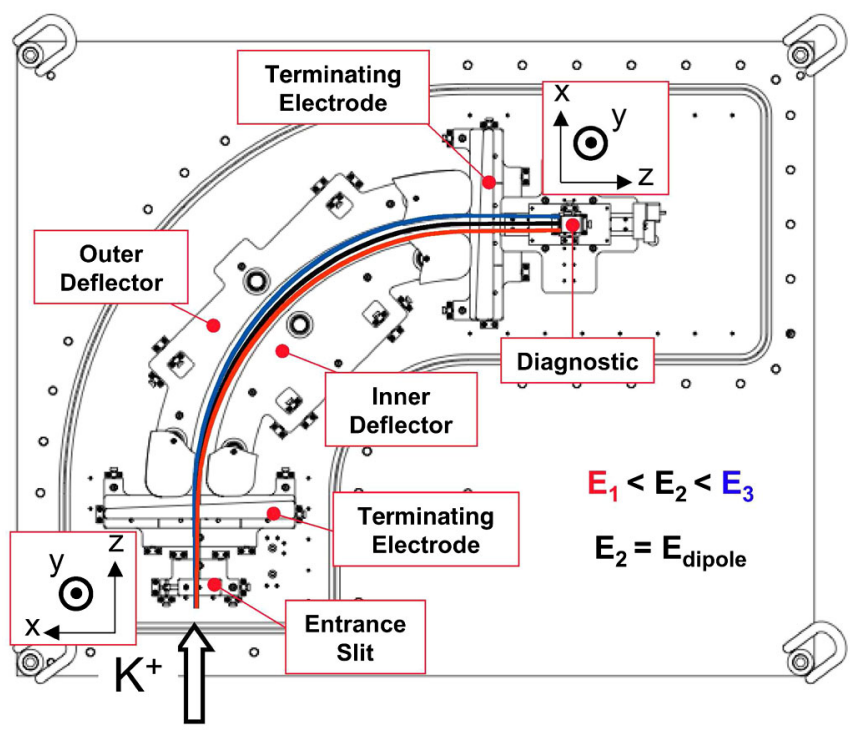

FIG. 4. Schematic of the ion trajectories though the spectrometer. Ions with energy greater than the dipole $\left(E_{3}\right.$, blue) are shown with a larger radius of curvature and ions with energy less than the dipole $\left(E_{1}\right.$, red $)$ are shown with a smaller radius of curvature. The coordinate system is shown at the entrance and exit of the spectrometer.

radius of curvature through the spectrometer $\left(E_{3}\right)$ and will have a positive first moment on the $x$ axis at the diagnostic plane $\left(+\left\langle x_{o}\right\rangle\right)$, where $\left\langle x_{o}\right\rangle=0$ is the center of the diagnostic plane. Ions with energy less than the dipole will follow a smaller radius of curvature through the spectrometer $\left(E_{1}\right)$ and will have a negative first moment on the $x$ axis $\left(-\left\langle x_{o}\right\rangle\right)$.

Measurements were made optically at the focal plane with a $100-\mu \mathrm{m}$ thick alumina scintillator, similar to that described in Refs. [13,14,20]. An image intensified gatedCCD camera captured the beam-induced light emission. The transverse resolution was $0.07 \mathrm{~mm} /$ pixel, which is better than the resolution of the spectrometer relative to the slit configuration used $(0.1 \mathrm{~mm})$ for these measurements. Consecutive measurements were made at different times throughout the $3 \mu \mathrm{s}$ beam pulse to determine the narrowest transverse distribution or energy profile. Through inspection it was determined that a 500 ns gate time was necessary to make a measurement with significant $S / N$ and sufficient due to the monochromaticity of the beam (shown below). The gate which captured the narrowest energy profile was delayed to $t=5.75 \mu \mathrm{s}$, where $t=0$ is the extraction time of beam from the diode. This corresponded to the same time in the middle of the $3 \mu$ s beam pulse where simultaneous focusing measurements were made in Ref. [21].

A demonstration of the displacement of the ions at the focal plane is shown in Fig. 5. The measurements were taken at the time frame selected above and the dipole field was varied over a range that corresponded to $286 \leq$ $E_{\text {beam }} \leq 292 \mathrm{keV}$, where $E_{\text {beam }}$ is the ion kinetic energy 


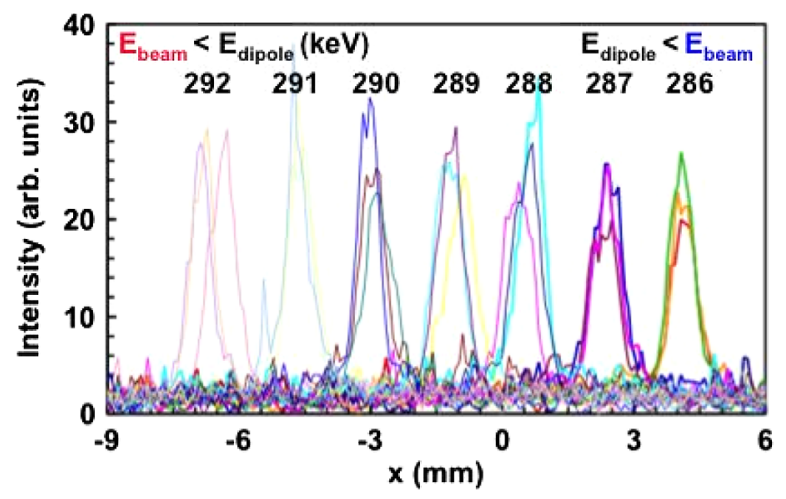

FIG. 5. Displacement of energy profiles transported through the spectrometer about the focal plane. All profiles are transverse slices of the beam integrated over a $500 \mathrm{~ns}$ gate in the middle of the $3 \mu$ s pulse projected onto the $x$ axis at the focal plane. The equivalent energy on the dipole in $\mathrm{keV}$ is labeled in black.

along the central trajectory of the spectrometer. The resulting energy profiles were measured with displacements about the $x$ axis. When $E_{\text {dipole }}>289 \mathrm{keV}$, where $E_{\text {dipole }}=$ $20 \mathrm{eV} / V \cdot V_{\text {dipole }}$ [Eq. (1)], the energy profiles typically had a $-\left\langle x_{o}\right\rangle$ because the beam energy was lower than the dipole energy. The opposite was the case when $E_{\text {dipole }}<289 \mathrm{keV}$. The relation of the position of the energy profile to the energy of the beam is given in Eq. (7), where a $1 \mathrm{keV}$ change in energy for a $300 \mathrm{keV}$ beam is equivalent to a movement of $1.67 \mathrm{~mm}$ at the focal plane.
The resulting ensemble of distributions was used to determine if there was an effective increase or decrease in the dipole radius from the designed $90^{\circ}$ bend. A fiducial on the scintillator was used for reference to the ideal centerline. After relating each of the measured distributions to the fiducial on the scintillator and taking a first moment of the ensemble of distributions it was determined $\left\langle x_{o}\right\rangle=-1.65 \mathrm{~mm}$ as shown in Fig. 5. This offset translated to an additional $9.4 \mathrm{mrad}\left(0.54^{\circ}\right)$ angle added to the designed $90^{\circ}$ bend. This correction was applied when determining the energy of the bunch for optical measurements.

From this data, one can also see there is a variation in position of the energy profile for an individual dipole setting. For all of the dipole settings in this ensemble of distributions, the energy of the beam at this particular time slice (500 ns gate in the middle of the $3 \mu$ s beam pulse) varied $\pm 0.24 \mathrm{keV}$.

\section{LONGITUDINAL TEMPERATURE MEASUREMENTS}

Transverse beam distributions [Fig. 6(a)] were transmitted to the diagnostic plane by bending the beam propagation axis $90^{\circ}$ using a curved electric field [Figs. 3 and 4]. The transverse distribution provides a measurement of longitudinal temperature of the beam, the width $\Delta x$ is a function of the geometry of the spectrometer and the energy spread $\Delta E$ of the beam:
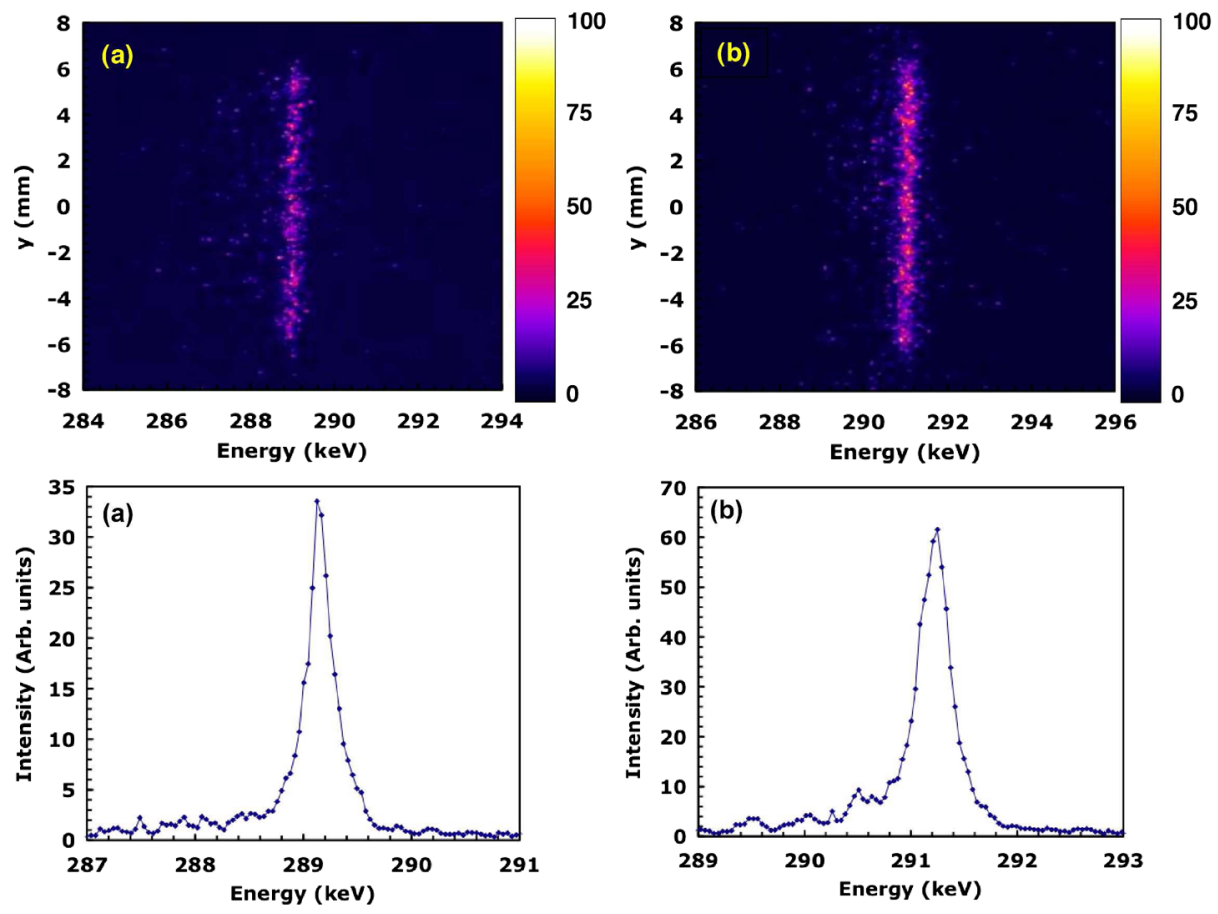

FIG. 6. Top row: Transverse slices of the beam integrated over a 500 ns gate in the middle of a $3 \mu$ s pulse. Bottom row: Intensity of the transverse slices projected onto the energy axis for: (a) an unneutralized beam; and (b) a beam neutralized by the FEPS (note scale differences). 


$$
\Delta x=\frac{a \Delta E}{2 E_{o}}(1+M)
$$

where $E_{o}$ is the particle energy that is transported through the center of the spectrometer. A $\Delta E=1 \mathrm{keV}$ for a $300 \mathrm{keV}$ beam is equivalent to $\Delta x=1.67 \mathrm{~mm}$ at the focal plane. This relation is also used to determine the energy of the beam for optical measurements. The energy spread is shown in the bottom row of Fig. 6.

Recall the beam passes through a $0.1 \mathrm{~mm}$ wide $\times$ $5 \mathrm{~mm}$ high slit plate and the measured transverse emittance is $14 \mu \mathrm{m}$ [14]. Beam expansion due to transverse emittance is small in the vertical direction and is confirmed by the height of the measured distribution in the top row of Fig. 6. Beam expansion is also negligible in the horizontal direction because of the electrostatic focusing of the beam as it propagates through the spectrometer. Without the transverse electric field that steers the beam longitudinally, the transverse distribution would expand to tens of centimeters in the horizontal direction. The measured profiles in Fig. 6 have a FWHM of $\sim 0.5 \mathrm{~mm}$ or $300 \mathrm{eV}$.

The distributions from the data acquired above were used to measure the longitudinal temperature. As explained above, the width $\Delta x$ of the transverse distribution measured at the diagnostic plane is a function of the geometry of the spectrometer and the energy spread $\Delta E$ of the beam. Assuming the longitudinal distribution is a $1 D$ Maxwellian, $\Delta E$ can be used to calculate the longitudinal temperature of the beam $T_{z}$ :

$$
T_{z}=\frac{(\Delta E)^{2}}{2 E_{o}} .
$$

Measurements were made of the $\mathrm{K}^{+}$ion beam itself and with the space-charge neutralized upstream of the spectrometer by the FEPS. Measurements of the $\mathrm{K}^{+}$ion beam itself displayed a $T_{z}=2.1 \pm 1.2 \times 10^{-2} \mathrm{eV}$. Measurements of the neutralized beam showed a slightly warmer temperature, $T_{z}=4.7 \pm 2.8 \times 10^{-2} \mathrm{eV}$. The beam distribution of the neutralized case has a current density 4 times greater than the unneutralized case upon entrance to the spectrometer; however this is still well below the $100 \mu \mathrm{A}$ limit, where space charge begins to play a role. Two examples of the transverse distributions measured and the integrated profiles are shown in Fig. 6. It is worth noting that an integrated source to target simulation calculated $T_{z}=2.0 \times 10^{-2} \mathrm{eV}$ [22]. This was done with a particle in cell code called large scale plasmas (LSP) [23].

Measurements were made with narrower time gates (time slices) to verify that the longitudinal temperatures were not intrinsically broadened due to longer gate times. Longitudinal phase space measurements in the next section also verify the time slice measured (middle of the $3 \mu \mathrm{s}$ beam pulse) is monochromatic and has a consistent $T_{z}$.

\section{LONGITUDINAL PHASE SPACE MEASUREMENTS}

Two methods were used to measure the longitudinal phase space of the beam. The first method was performed optically with an image intensified streak camera and the second with two slits.

\section{A. Optical measurements}

The transverse beam distributions from optical measurements with the scintillator were measured as a function of time with an image intensified streak camera. In principle, the streak camera has the ability to resolve the longitudinal phase space from the transverse beam distribution at the optical plane for a single shot. We used a Hamamatsu streak C7700 [24] which has a temporal resolution of 5 ps and a $1344 \times 1024 \mathrm{CCD}$ pixel array. The transverse resolution of the measurements on the CCD $(0.072 \mathrm{~mm} / \mathrm{pix})$ was nearly identical to the optical measurements discussed in Sec. IV.

We have taken measurements with the slit of the streak camera set to integrate the beam over 250 ns slices throughout the $3 \mu \mathrm{s}$ beam pulse. This cuts down the amount of light collected from previous measurements with the image intensified gated-CCD camera by a factor of 4 . This is because we are integrating over half as long of a time window and the slit is only capturing $4 \mathrm{~mm}$ of the 10-mm high beam distribution (Fig. 6). An average longitudinal phase space distribution from 10 streaked images with a dipole setting of $289 \mathrm{keV}$ is shown in Fig. 7. The beam energy fluctuations $(\langle\Delta E\rangle=240 \mathrm{eV})$ from shot to shot can induce a false widening to the width of the intrinsic distribution. The light collection efficiency was low for these measurements and should be improved in the future to maximize the use of the CCD. The CCD for this streak camera is $>4$ times larger than CCD on the PI-Max

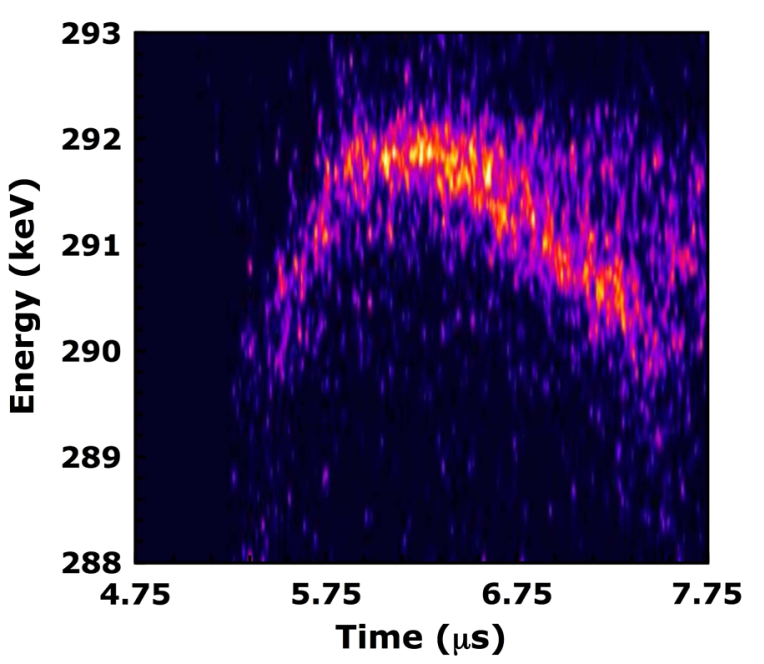

FIG. 7. Longitudinal phase space distribution of the NDCX beam with a $3 \mu$ s pulse streaked in time. 
used for images described above, so a closer coupling via additional focusing lenses and a fiber optic bundle is necessary.

A sloped region near the head and at the tail of the beam is observed (Fig. 7). The slope at the head of the beam is $4 \mathrm{kV} / \mu \mathrm{s}$ from $5.25-5.75 \mu \mathrm{s}$. There is a flat region where the beam energy was relatively constant from 5.75 to $>6.25 \mu \mathrm{s}$. This is the relevant time frame at which the longitudinal temperature measurements (presented in Secs. IV and V) were made, confirming this is the best operating point for axial compression for this pulse length. After the monochromatic region in phase space, the beam energy has a negative slope $\sim 2 \mathrm{kV} / \mu \mathrm{s}$ from $t \geq 6.25 \mu \mathrm{s}$. We do expect the longitudinal phase space to have this structure; there is a finite rise time, flattop, and fall time of the extraction voltage in the diode as indicated in the next section.

\section{B. Slit and Faraday collector measurements}

Another method of measuring the longitudinal phase space is made with a slit and a Faraday collector (slit cup) at the focal plane of the spectrometer. This method is similar to that used for transverse phase space measurements $[13,14]$. The upstream slit is the same as described above $(0.1 \mathrm{~mm} \times 5 \mathrm{~mm})$ and the downstream slit was increased to $0.1 \mathrm{~mm} \times 10 \mathrm{~mm}$ to compensate for beam expansion due to the transverse emittance in the vertical (nonbend) plane. This measurement is not as effective as the optical case because it consists of an ensemble of signals taken at various dipole energies, making the width of the distribution subject to intrinsic widening due to energy variations from shot to shot.

Step sizes for the dipole in these measurements could be as small as the resolution of the spectrometer $(60 \mathrm{eV})$ but larger steps $(200 \mathrm{eV})$ were taken due to minimum energy variation of the beam from shot to shot $(\langle\Delta E\rangle=240 \mathrm{eV})$. The slit cup generally measured tens of millivolts $(\sim \mu \mathrm{A}$ of current) and the $S / N$ was close to 10 when configured to measure secondary electrons leaving the collector and using an amplifier. The measured distribution for a $2 \mu \mathrm{s}$ beam pulse using this technique is shown in Fig. 8. The pulse length for these measurements was shortened to eliminate electrical noise in the circuit due to the Marx crowbar (explained below). The $S / N$ was already close to 10 when configured to measure secondary electrons leaving the collector and using an amplifier, so any additional noise (i.e. from the crowbar) made the signals difficult to resolve.

An identical slope of $4 \mathrm{kV} / \mu \mathrm{s}$ is measured at the head of the beam for this setup when compared to the optically measured longitudinal phase space distribution (Figs. 7 and 8 ). There is also a sharp structure at the head of the beam near $5.6 \mu \mathrm{s}$. This could be real or may be an intrinsic effect due to the variation of the beam energy from shot to shot $(\langle\Delta E\rangle=240 \mathrm{eV})$. Afterwards there is also a monochro-

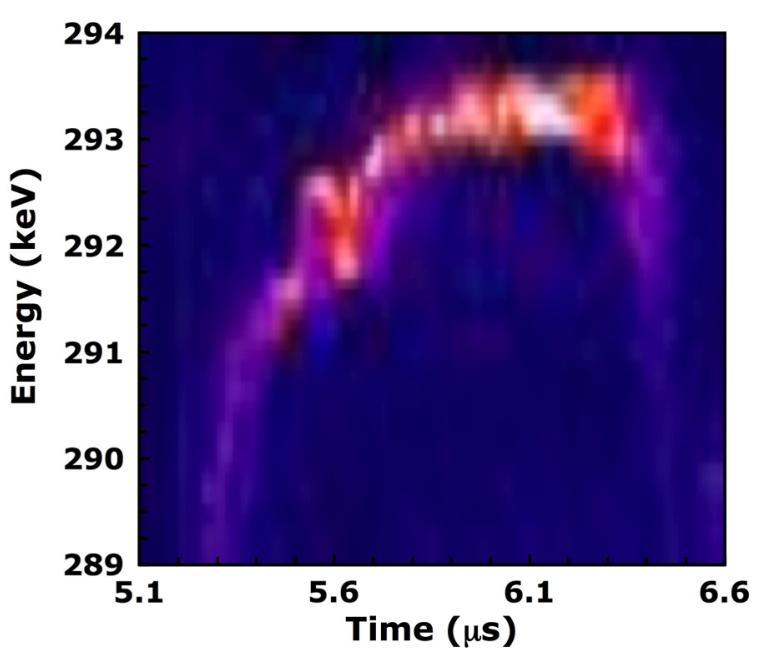

FIG. 8. Longitudinal phase space distribution of the NDCX beam with a $2 \mu$ s pulse measured with two slits.

matic region where the beam energy was relatively constant for about $500 \mathrm{~ns}$, which was also seen in the optical measurements.

\section{CORRELATION OF THE LONGITUDINAL MEASUREMENTS WITH THE MARX VOLTAGE}

Each of the methods of longitudinal measurement discussed in this section were compared to the frequently measured Marx voltage waveform, which is used to extract the beam from the diode. A Marx generator is a pulsed power circuit composed of a string of parallel capacitors and resistors connected in series by spark gaps [25]. The circuit can be configured with multiple stages designed for a desired voltage and pulse length. They are commonly used in the accelerator, pulsed power, and plasma physics communities for pulsed high voltage applications. The Marx generator on the NDCX was a 500-kV Marx with pulse lengths variable from $400 \mathrm{~ns}-$ tens of $\mu \mathrm{s}$.

First a detailed comparison was made with the optical measurements of the energy profiles measured in Sec. IV. The time used for the optical measurements of the energy profiles corresponded to about $1.25 \mu \mathrm{s}$ after the Marx was triggered. A strong correlation is shown in Fig. 9 with the shot to shot variation of the Marx and the measured beam energy at this time. Although the actual values do not match up exactly, due to possible discrepancies in the capacitive divider used to measure the Marx voltage, the general trend is the same. Each time the voltage varies in the Marx, a corresponding change in the beam energy is measured. The average Marx voltage for this particular data set was $304.10 \pm 1.78 \mathrm{kV}$ and the beam energy was $290.26 \pm 1.03 \mathrm{keV}$, each varied $<1 \%$. This energy variation is a factor of 5 greater than what was determined for a larger data set in Sec. V for unknown reasons. It is worth noting from this comparison that the beam energy measured by the EEA is $4.55 \pm 0.57 \%$ lower than the voltage 


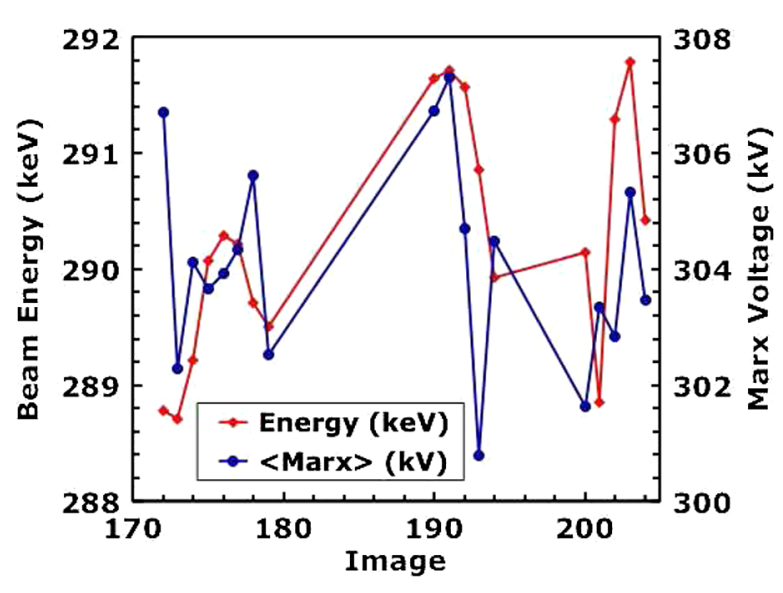

FIG. 9. Correlation of the average Marx voltage (blue for $1.25<$ Time $(\mu \mathrm{s})<1.75)$ with individual measurements of the energy profile (red) made with the CCD camera (note scale differences).

monitored by the Marx capacitive divider. This complements measurements made with the same Marx bank and capacitive divider in 2004 on the Neutralized Transport Experiment [26]. These measurements were made with a resistive divider and a time of flight (TOF) technique. Each displayed a slightly lower trend in energy than monitored by the capacitive divider voltage. The resistive divider voltage was $1.73 \pm 0.56 \%$ less than the capacitive divider voltage and the energy calculated by TOF was $4.28 \pm$ $0.94 \%$ less than the capacitive divider voltage.

Next the first moments of the longitudinal phase space distributions $(\langle E\rangle)$ from Figs. 7 and 8 were compared to the average Marx waveforms from the series of shots taken to obtain the distributions. After examining Fig. 10 you can see the differences between the Marx waveform and $\langle E\rangle$; in both cases where there is a steep rise at the beginning of the Marx pulse $(\sim 20 \mathrm{kV} / \mu \mathrm{s})$ and a steady slope down of $4 \mathrm{kV} / \mu \mathrm{s}$ after the peak; for the $3 \mu$ s pulse this continues even longer. This is in slight disagreement with the $\langle E\rangle$ measured in both cases. As described in Sec. VI, the head of the beam in the longitudinal phase space distributions
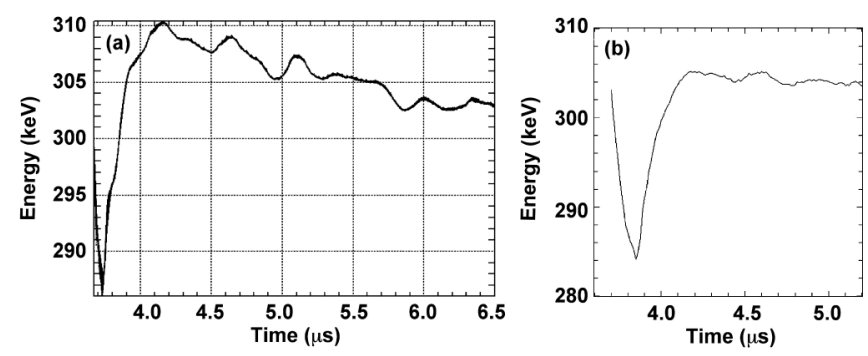

FIG. 11. Longitudinal phase space distributions calculated in: (a) LSP; and (b) WARP.

has a slope of $4 \mathrm{kV} / \mu \mathrm{s}$ for $\sim 500 \mathrm{~ns}$, then there is a monochromatic region for $500 \mathrm{~ns}$ or greater. For the $3 \mu \mathrm{s}$ pulse there is an average negative slope of $2 \mathrm{kV} / \mu \mathrm{s}$ after the monochromatic region. This structure in the longitudinal phase space is expected due to the finite rise time, flattop, and fall time of the extraction voltage in the diode. However, these results indicate there is a slight sag in the capacitive divider response for the Marx waveform. It is also worth noting the average Marx voltage is nearly $2 \mathrm{kV}$ lower for the optical data set in comparison to the data set taken with 2 slits. The measured beam energy is also slightly lower for the optical data set which may help explain the fluctuation in the measured error relative to Marx voltage noted above.

Numerical simulations were also performed in WARP [27-30] and LSP [23] to model the longitudinal dynamics. Both PIC methods used the experimental Marx waveform as an input to calculate the beam dynamics. The calculated distributions show a longitudinal space-charge effect near the head of the beam, $t<4 \mu \mathrm{s}$, and a distribution that agrees well with the Marx waveform after the head (Fig. 11). Both calculations show a $16 \mathrm{kV}$ drop over $100 \mathrm{~ns}$ for the leading particles in the head of the distribution. Then a $22 \mathrm{kV}$ increase in energy over $300 \mathrm{~ns}$ $(\sim 73 \mathrm{kV} / \mu \mathrm{s})$ follows. This energy variation due to space charge was not resolved in the longitudinal phase space measurements, which only show a $2 \mathrm{kV}$ rise over $500 \mathrm{~ns}$ at the head.
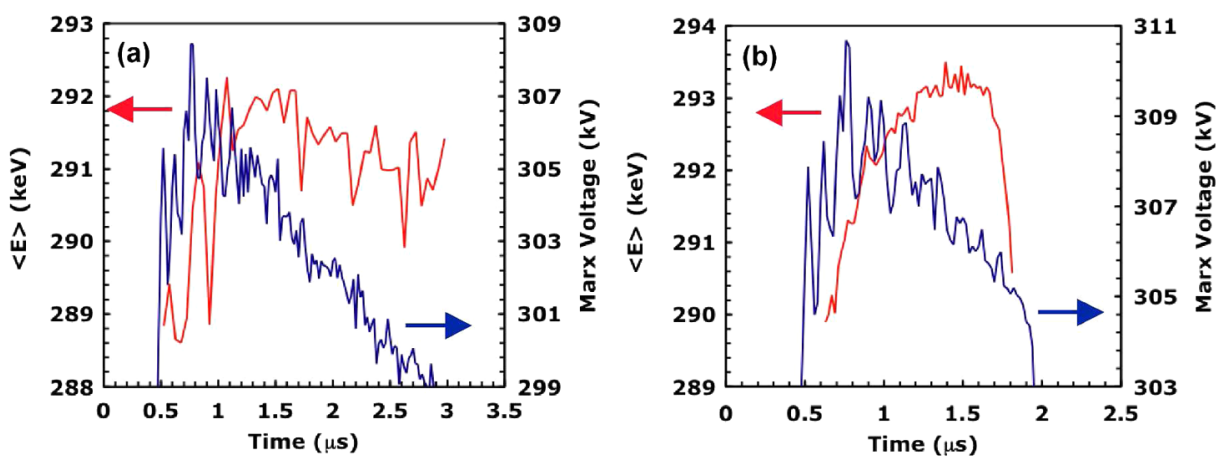

FIG. 10. Correlation of the Marx voltage (blue) with the $\langle E\rangle$ (red) resolved from longitudinal phase space measurements with: (a) the streak camera for a $3 \mu$ s pulse; and (b) the slit cup for a $2 \mu$ s pulse (note scale differences). 
The space-charge effect at the head of the beam was most likely not measured because the envelope of these particles was not tuned to transport these particles without loss to the entrance of the spectrometer. Recall there is a $180 \mathrm{~cm}$ drift space from the end of the transport lattice to the entrance of the spectrometer. The longitudinal spacecharge effect causes an overtaking or snowplow effect at the head of the beam, increasing the current along the axis of propagation making it more susceptible to scraping or beam loss. In addition, the current density of the head distribution at the entrance slit to the spectrometer may be below the detection threshold of $\sim 0.1 \mathrm{~A} / \mathrm{m}^{2}$.

\section{CONCLUSIONS}

We effectively measured the longitudinal temperature and phase space of an intense ion beam. Measurements of the longitudinal temperature during the relevant time of axial compression [21] indicate $T_{z}=2.1 \pm 1.2 \times$ $10^{-2} \mathrm{eV}$ for the $\mathrm{K}^{+}$ion beam itself and $T_{z}=4.7 \pm 2.8 \times$ $10^{-2} \mathrm{eV}$ for the $\mathrm{K}^{+}$ion beam neutralized by the FEPS. A recent source to target simulations in LSP calculates $T_{z}=2 \times 10^{-2} \mathrm{eV}$ in good agreement with the measurement presented here [22], contrary to the previous assumptions for simulations. These measurements set a lower bound on the minimum longitudinal temperature achievable on NDCX in addition to a lower bound on the minimum pulse widths achievable for axial compression. This provides additional thrust to improve the temporal resolution of the fast diagnostics currently used to measure axial compression (i.e. the fast pinhole Faraday cup and scintillator material).

The Marx voltage waveform appears to provide a qualitative measurement of the beam energy, although comparisons indicate the beam energy measured by the EEA is $4.55 \pm 0.57 \%$ lower than the voltage monitored by the Marx capacitive divider. This is in agreement with past measurements [26]. Longitudinal phase space measurements show a slight difference between the longitudinal dynamics and the Marx waveform. Calculations in WARP and LSP show a space-charge effect at the beam head that was not measured. The calculations also reproduce the slopes measured in the Marx waveform giving slight disagreement between the calculations and measurements of the longitudinal phase space. This indicates the Marx waveform does not represent the dynamics of the longitudinal beam bunch from head to tail with $100 \%$ accuracy. In addition the finite structure of the space-charge effect at the head of the beam is difficult to measure because the current density of this portion of the beam is too low.

\section{ACKNOWLEDGMENTS}

This work was supported by the Director, Office of Science, Office of Fusion Energy Sciences, of the U.S. Department of Energy under Contract No. DE-AC02-
05CH11231. Special thanks to Tak Katayanagi, Michael Dickinson, Cory Lee, Wayne Greenway, Craig Rogers, and Andy Faltens for their technical support.

[1] R. Tikhoplav et al., in Proceedings of LINAC, Lübeck, Germany, 2004, p. 147 [http://accelconf.web.cern.ch/ AccelConf/104/PAPERS/MOP46.PDF].

[2] Bertsche et al., in Proceedings of the Particle Accelerator Conference, Vancouver, Canada, 2009 (IEEE, Piscataway, NJ, 2009), p. 2346.

[3] Rihaoui et al., in Proceedings of the Particle Accelerator Conference, New York, NY, 2011 (IEEE, New York, 2011), p. 1858.

[4] M. Tanabe et al., Appl. Phys. Express 1, 028001 (2008).

[5] M. Steck, K. Beckert, H. Eickhoff, B. Franzke, F. Nolden, H. Reich, B. Schlitt, and T. Winkler, Phys. Rev. Lett. 77, 3803 (1996).

[6] C. Dimopoulou et al., in Proceedings of the European Particle Accelerator Conference, Genoa, Italy, 2008 (EPS-AG, Genoa, Italy, 2008), p. 3470.

[7] M. A. Leitner, C. M. Celata, E. P. Lee, B. G. Logan, W. L. Waldron, S.S. Yu, and J. J. Barnard, Nucl. Instrum. Methods Phys. Res., Sect. A 544, 315 (2005).

[8] B. G. Logan et al., in Proceedings of the Workshop on Accelerator-Driven High Energy Density Physics (2004) Appendix A1, held at LBNL; Reports No. LBNL-57518 and No. UCRL-PROC-212000, 2005 [http://hifweb.lbl .gov/public/hedpworkshop/toc.html].

[9] A. Friedman et al., in Proceedings of the International Computational Accelerator Physics Conference, San Francisco, CA, 2009, p. 157 [http://epaper.kek.jp/ ICAP2009/html/author.htm].

[10] A. Friedman et al., Phys. Plasmas 17, 056704 (2010).

[11] P. K. Roy et al., Phys. Rev. Lett. 95, 234801 (2005).

[12] P. A. Seidl et al., Nucl. Instrum. Methods Phys. Res., Sect. A 577, 215 (2007).

[13] J.E. Coleman et al., Phys. Rev. ST Accel. Beams 11, 050101 (2008).

[14] J.E. Coleman, P. A. Seidl, J. L. Vay, A. W. Molvik, and W. M. Sharp, Phys. Rev. ST Accel. Beams 11, 050103 (2008).

[15] F. M. Bieniosek et al., J. Phys. Conf. Ser. 244, 032028 (2010).

[16] R.E. Warren, J.L. Powell, and R. G. Herb, Rev. Sci. Instrum. 18, 559 (1947).

[17] http://www.glassmanhv.com/ByWattage/wr_series.shtml.

[18] http://www.rossengineeringcorp.com/high_accuracy.htm.

[19] http://www.tek.com/oscilloscope/tds3000.

[20] F. M. Bieniosek et al., Nucl. Instrum. Methods Phys. Res., Sect. A 544, 268 (2005).

[21] J.E. Coleman et al., in Proceedings of the Particle Accelerator Conference, Albuquerque, NM, 2007 (IEEE, New York, 2007), p. 3516 [http://www.JACoW.org/].

[22] D. R. Welch, J. E. Coleman, P. A. Seidl, P. K. Roy, E. Henestroza, E. P. Lee, A. B. Sefkow, E. P. Gilson, T. C. Genoni, and D. V. Rose, Phys. Rev. ST Accel. Beams 11, 064701 (2008).

[23] LSP is a software product of ATK Mission Research [http:// www.mrcwdc.com/LSP/index.html]. 
[24] http://sales.hamamatsu.com/en/products/system-division/ ultra-fast/streak-systems/part-c7700.php.

[25] E. Marx, Verfahren zur Schlagprüfung von Isolatoren und anderen elektrischen Vorrichtungen (Method for breakdown tests of isolators and other electric devices). German Patent No. 455933, 1923.

[26] E. Henestroza, S. Eylon, P. K. Roy, S.S. Yu, F. M. Bieniosek, D. B. Shuman, and W. L. Waldron, Phys. Rev. ST Accel. Beams 8, 052801 (2005).
[27] A. Friedman, D. P. Grote, and I. Haber, Phys. Fluids B 4, 2203 (1992).

[28] D. P. Grote, A. Friedman, and I. Haber, AIP Conf. Proc. 391, 51 (1997).

[29] D. P. Grote, A. Friedman, I. Haber, W. Fawley, and J. L. Vay, Nucl. Instrum. Methods Phys. Res., Sect. A 415, 428 (1998).

[30] D.P. Grote, A. Friedman, J.L. Vay, and I. Haber, AIP Conf. Proc. 749, 55 (2005). 